Perspectiva Geográfica ISSN 0123-3769 (Impreso) - 2500-8684 (En línea)

Vol. 21 N. ${ }^{\circ} 2$ de 2016

Julio - Diciembre pp. 321-344

\title{
Potencial para la conservación de la geodiversidad de los paisajes del Estado de Michoacán, México ${ }^{1}$
}

\author{
Potential for Conservation of Geodiversity of the Landscape of \\ the State of Michoacan, Mexico
}

\section{Luis Giovanni Ramírez Sánchez $^{2}$ \\ Ángel G. Priego $\sim$ Santander ${ }^{3}$ Manuel Bollo Manent ${ }^{4}$ Danays del Carmen Castelo Agüero $^{5}$}

Para citar este artículo utilice el nombre completo así:

Ramírez-Sánchez, L. G., Priego-Santander, A. G., Bollo, M. y Castelo-Agüero, D. C. (2016). Potencial para la conservación de la geodiversidad de los paisajes del Estado de Michoacán, México. Perspectiva Geográfica, 21(2), 321-344. doi: 10.19053/01233769.5856

\section{Resumen}

La heterogeneidad del paisaje varía en el espacio dependiendo de la proporción entre polígonos y unidades tipológicas. La alta variabilidad del

1 Este artículo es resultado de la tesis de Doctorado en Geografía, realizada por el primer autor en el Centro de Investigaciones en Geografía Ambiental UNAM-Morelia.

2 Joven Investigador Cátedras CONACyT-El Colegio Mexiquense A. C. Iramirez@cmq.edu.mx

3 PhD en Geografía. Investigador titular "A", Centro de Investigaciones en Geografía Ambiental UNAM-Morelia. apriego@ ciga.unam.mx

$4 \mathrm{PhD}$ en Geografía. Técnico Académico titular "A", Centro de Investigaciones en Geografía Ambiental UNAM-Morelia. mbollo@ciga.unam.mx

5 Estudiante de Doctorado en Geografía, Centro de Investigaciones en Geografía Ambiental UNAM-Morelia. adanays@ pmip.unam.mx 
espacio geográfico que posee el estado de Michoacán le confiere una elevada connotación ecológica, lo cual sugiere valores importantes de geodiversidad. Para conocer la heterogeneidad de cada geocomplejo se computó el número de polígonos, así como el número de parajes simples que contenía cada uno de estos y se aplicaron indicadores de complejidad tipológica, corológica, diversidad máxima y de Mclntosh, así como riqueza de paisajes. Se calculó un valor único para cada unidad de paisaje con el propósito de elaborar un mapa unificado del potencial natural para la conservación de la geodiversidad. Para ello se calculó un valor promedio entre todos los indicadores por unidades de paisajes y se distinguieron cinco categorías para la conservación de la geodiversidad. Los resultados principales muestran que en la Meseta Purépecha se encuentran potenciales valiosos para la conservación de la geodiversidad.

Palabras clave: geodiversidad, heterogeneidad geoecológica, Michoacán, paisajes.

\begin{abstract}
Landscape heterogeneity varies in the space depending on the ratio between polygons and typological units. The high variability of the geographical area of Michoacan state has conferred a high ecological connotation, suggesting important values of geodiversity. To better understand the landscape heterogeneity, complex polygon count was computed and the number of sites containing each of these polygons. For each polygon the indexes such as typological complexity, chorological, relative richness of ecosystem and maximum diversity were applied. A single value was calculated for each landscape in order to develop a unified map of the natural potential for conservation of geodiversity. To this end an average value among all indicators of the landscapes units was calculated and five categories were distinguished. The results show that in the Purepecha Plateau region valuable potentials for the conservation of geodiversity were found.
\end{abstract}

Keywords: Geodiversity, Geoecological Diversity, Michoacan, Landscapes. 


\section{Introducción}

La heterogeneidad del hábitat es fundamental para el funcionamiento de los ecosistemas. Los ecólogos recientemente han comenzado a explorar cómo los cambios en la heterogeneidad del hábitat pueden influir en el funcionamiento del ecosistema al afectar importantes procesos como la producción primaria, la descomposición o el ciclo de nutrientes (Poggiale et al., 2005; Yi, et al., 2009; Peng, et al., 2013; Plexida, et al., 2014). La heterogeneidad del hábitat, además de tener una gran influencia en importantes procesos bióticos y abióticos, tales como alteración del paisaje y el movimiento de los organismos (Turner, 2005; Turner, et al., 2014), puede estar relacionada con la diversidad de especies, la resistencia y la función del ecosistema (Huston, 2014).

La heterogeneidad ecológica es un concepto acuñado en los primeros pasos de la historia de la Ecología (McIntosh, 1991). Milne (1991) define la heterogeneidad ecológica como la complejidad resultante de las interacciones entre la distribución de los factores ambientales y la respuesta diferencial de los organismos a esos factores. Dicho en otras palabras, los organismos viven en hábitats que son sumamente heterogéneos, tanto en espacio como en tiempo (Stewart, et al., 2000). Sin embargo, la heterogeneidad ecológica frecuentemente va asociada a la escala en que se mide; esto quiere decir que los procesos e interacciones que se aprecian a distintas escalas de observación pueden no coincidir o incluso, ser contradictorios. La consecuencia principal de esta afirmación es que los resultados obtenidos de una cuestión ecológica en particular pueden depender fuertemente de la escala a la cual el estudio es llevado a cabo (Turner, et al., 2001).

El concepto de heterogeneidad del paisaje se usa comúnmente en Ecología del Paisaje para referirse a la heterogeneidad de la vegetación o del uso del suelo (Kotliar \& Wiens, 1990; Forman, 1995; Bowler \& Benton, 2005; Ganzei \& Ivanov, 2012; Cruz-García, et al. 2015), refiriéndose a la agregación o a la dispersión de los parches de los tipos de cobertura y a su contraste; o sea, al grado de diferencia entre los parches y la matriz (Uezu, et al., 2005; Schippers, et al., 2015).

El análisis de patrones de la estructura del paisaje o la cuantificación 
de la heterogeneidad espacial es crucial para entender los procesos ecológicos subyacentes y su dinámica (Scheiner, 1992; McGarigal \& Marks, 1995; Stein \& Kreft, 2014). La heterogeneidad, como concepto sistémico, puede definirse como el grado de variabilidad espacial de alguna propiedad dentro de un sistema (Li \& Reynolds, 1995; Morgan \& Gergel, 2010).

La Geoecología, como ciencia geográfica, utiliza el enfoque paisajístico (policéntrico) o geoecológico, y toma como base del análisis espacial a los paisajes físico-geográficos. La Geoecología realiza investigaciones desde una perspectiva de análisis espacial holístico y a escalas espaciales relativamente más amplias que la Ecología Biológica del pasado. La Geoecología tiene funciones y objetivos específicos: proporciona bases técnicas adecuadas para la planificación y el uso de la tierra (Lavrinenko, 2012; Priego-Santander et al., 2013) o de manera más práctica, trabaja en la solución de problemas de ordenamiento y manejo de los recursos naturales (Haines-Young, 1999; Boone \& Krohn, 2000; Bastian, 2001; Moss, 2001; Antrop, 2007; Termorshuizen \& Opman, 2009). Además, juega un rol importante en la definición de políticas de conservación y gestión de la biodiversidad y la geodiversidad (Dowling, 2013; Zaragoza, et al., 2013; Matthews, 2014; Merino, et al., 2015). Esto cobra mayor importancia debido a los acelerados procesos de pérdida de la diversidad biológica que hoy se producen a nivel mundial (Opdam, et al., 2003; Ramírez-Sánchez, 2009).

Las unidades espaciales para las investigaciones en Geoecología constituyen un sistema de unidades taxonómicas vinculado a la escala de representación cartográfica, que va desde el nivel regional (1:250.000 o escalas menores), a la escala local (1:250.000 o escalas mayores). El nivel local tiene cuatro niveles taxonómicos: la localidad físico-geográfica, que está formada en su interior por comarcas o parajes físico-geográficos complejos, los cuales contienen subcomarcas o parajes físico-geográficos simples y, a su vez, se componen de facies. La representación de estos taxones en un mapa muestra la diferenciación de los paisajes en un espacio dado (estructura horizontal de los paisajes), y permite identificar la heterogeneidad de los mismos (Mateo, 2002; 2007). 
En la Geoecología, como ciencia geográfica, a la heterogeneidad del paisaje se le conoce como heterogeneidad geoecológica; esto de acuerdo con Rowe (1995) y Ganzei e Ivanov (2012) no es más que la complejidad de la estructura horizontal de los paisajes (geosistemas) en un territorio dado, resultado de la diferenciación espacial de los mismos y de sus componentes naturales. Estos están condicionados por la distinta composición en tipos y por el número de polígonos de cada clase con respecto a la unidad superior. De este modo, la heterogeneidad de un paraje físicogeográfico complejo ${ }^{6}$ (comarca físico-geográfica) está determinada por el número y variedad de parajes físico-geográficos simples que lo integran (subcomarcas físico-geográficas). La heterogeneidad geoecológica se determina según la escala de estudio a partir de las unidades taxonómicas del mapa de paisajes en cuestión, con la ayuda de un conjunto de indicadores como complejidad, diversidad, riqueza, abundancia, dominancia, entre otros.

La heterogeneidad geoecológica refleja el potencial natural de un

6 Los Parajes físico-geográficos complejos son unidades del nivel local de los paisajes. paisaje para la conservación de la geodiversidad. La geodiversidad es un concepto relativamente nuevo que ha tenido un bajo perfil hasta las dos últimas décadas, en las que su utilización por las ciencias geográficas crece rápidamente (Burek \& Prosser, 2008; Palacio, 2013; Dóniz-Páez y Becerra-Ramírez, 2015; García-Sánchez, et al., 2015). Actualmente, este concepto en Geografía es homólogo al de biodiversidad en el campo de las Ciencias Biológicas. Sin embargo, ambos conceptos han sido usados de manera desproporcionada, la biodiversidad ha sido definida formalmente, mientras que la geodiversidad aparece poco contemplada en los diferentes tratados nacionales e internacionales sobre conservación del patrimonio natural.

La geodiversidad se puede entender como una síntesis del paisaje, en la que se incluyen los elementos y procesos geológicos, hidrológicos, geomorfológicos, edáficos y climáticos (Alexandrowicz \& Kozlowski, 1999; Palacio, 2013; British Geological Survey, 2014; Lozano, 2015; Medina, 2015). Además, considera los entramados de relaciones entre elementos y procesos del medio físico (Sharples, 2002). 
De acuerdo con Kozlowski (2004), la definición más integradora de la geodiversidad es la que contempla la "variedad natural en la superficie terrestre, referida a los aspectos geológicos, geomorfológicos, suelos, hidrología, así como otros sistemas generados como resultado de procesos naturales (endógenos y exógenos) y la actividad humana" (Silva, et al., 2014, p. 834). Asimismo, Brilha (2016) menciona que, cuando los elementos constituyentes de la geodiversidad presentan un alto valor científico, estos se consideran patrimonio geológico o geopatrimonio.

La presente investigación se desarrolló en el Estado de Michoacán, tomando como base el mapa de los paisajes físico-geográficos de dicho Estado a escala 1:250.000, elaborado por Ramírez-Sánchez, et al. (2012). El objetivo principal consiste en conocer el potencial natural para la conservación de la geodiversidad de dicho territorio, a partir del análisis de la heterogeneidad geoecológica en las unidades de paisajes físico-geográficos, en términos de su riqueza y diversidad espacial.

Resulta de gran relevancia teórica y práctica realizar estudios territoriales a través de métodos de análisis que integren los enfoques ecológico y geográfico en una rama de conocimiento denominada Ecología del Paisaje o Geoecología (Hasse, 1986). Esto acontece, ante la latente necesidad de integrar a los estudios de ecosistemas terrestres enfoques científicos transdisciplinarios, los cuales ayuden a mejorar el entendimiento sobre las relaciones ecológicas de sus componentes (incluidos el medio físico-geográfico, el biótico y el antrópico).

\section{Materiales y Métodos}

\section{1. Área de estudio}

El Estado de Michoacán se encuentra en la región Oeste de la República Mexicana y se ubica entre los ríos Lerma y Balsas, el lago de Chapala y el Océano Pacífico; entre las coordenadas $17^{\circ} 55^{\prime} 15^{\prime \prime}$ y $20^{\circ} 24^{\prime} 10^{\prime \prime}$ de latitud N y $103^{\circ} 44^{\prime} 27^{\prime \prime}$ y $100^{\circ} 04^{\prime} 11$ " de longitud O. El Estado cubre una extensión de $58.370 \mathrm{~km}^{2}$ (INEGIDGG, 1999; INEGI, 2001) que representa alrededor de $3 \%$ de la superficie total del territorio nacional, ocupando el decimosexto lugar en extensión entre las 32 entidades del país. Tiene un litoral que se extiende a lo largo de $217 \mathrm{~km}$ sobre el Océano Pacífico (Figura 1). 


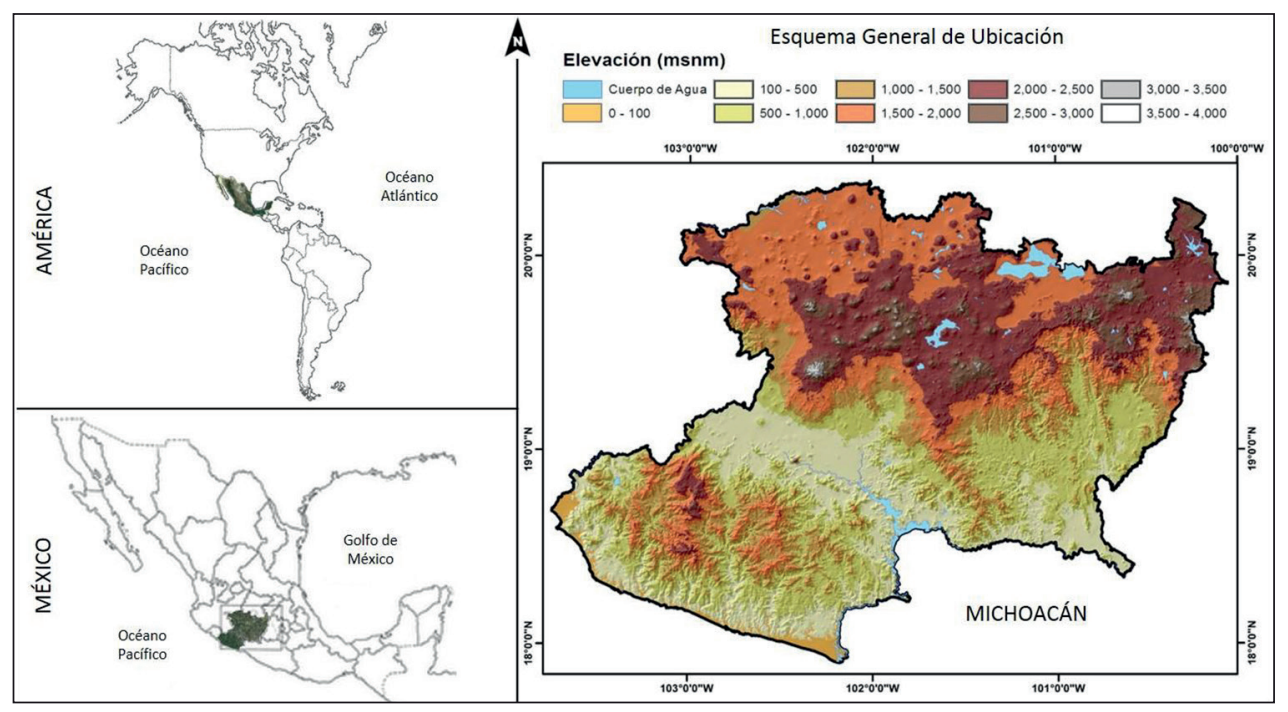

Figura 1. Esquema general de la localización del área de estudio

Fuente: Elaboración propia.

1.2. Paisajes físico-geográficos de gráficas. La clasificación tomó en Michoacán cuenta dos niveles taxonómicos representados en el mapa de paisa-

Para la clasificación se utilizó el jes; el paraje complejo y el paraje sistema de unidades taxonómicas simple, adoptándose como se obdel nivel local propuesto por Vidi- serva la nomenclatura de paraje en na (1970) (Tabla 1), que incluye las sustitución de comarca, propuesta localidades, comarcas simples (pa- por Aguirre-López (2010) por ser raje complejo), subcomarcas (pa- más comprensible para México.

rajes simples) y facies físico-geo- 
Tabla 1. Índices diagnósticos del paisaje natural y de sus partes morfológicas (estructura horizontal)

\begin{tabular}{|c|c|c|}
\hline $\begin{array}{l}\text { Índice diagnóstico } \\
\text { principal: complejidad de } \\
\text { la estructura horizontal del } \\
\text { geosistema }\end{array}$ & $\begin{array}{l}\text { Unidad } \\
\text { morfológica }\end{array}$ & $\begin{array}{l}\text { Índice diagnóstico complementario: factores } \\
\text { naturales conjugados (relieve, condiciones } \\
\text { litológicas, hidrológicas y de otro tipo) }\end{array}$ \\
\hline Paisaje elemental & Facie & $\begin{array}{l}\text { Caracterizado por la situación en los límites de } \\
\text { un mismo elemento del relieve (a veces en una } \\
\text { microforma del relieve), la misma composición } \\
\text { litológica de la roca superficial, un mismo } \\
\text { régimen de humedad del suelo y del manto, el } \\
\text { mismo subtipo del suelo y la biocenosis. }\end{array}$ \\
\hline $\begin{array}{l}\text { Paisaje de estructura de } \\
\text { un escalón, formado por } \\
\text { facies individuales }\end{array}$ & Eslabón & $\begin{array}{l}\text { Caracterizado por la situación en los límites } \\
\text { de una microforma del relieve, la misma } \\
\text { composición litológica, idéntico régimen de } \\
\text { humedad del suelo y del manto, el mismo subtipc } \\
\text { de suelo y la misma biocenosis. }\end{array}$ \\
\hline $\begin{array}{l}\text { Paisaje de estructura de } \\
\text { un escalón, formado por } \\
\text { facies individuales }\end{array}$ & $\begin{array}{l}\text { Sub-comarca } \\
\text { (Paraje simple) }\end{array}$ & $\begin{array}{l}\text { Caracterizado por la situación en un elemento de } \\
\text { la mesoforma del relieve. Es semejante en cuanto } \\
\text { al ingreso de calor y luz solar (exposición). } \\
\text { Tiene la misma correlación en los depósitos o } \\
\text { litología y de la capa de formación de suelos; el } \\
\text { mismo tipo de régimen de la humedad del manto } \\
\text { y de los suelos. Un mismo tipo de suelos y de } \\
\text { biocenosis. }\end{array}$ \\
\hline $\begin{array}{l}\text { Paisaje de estructura de } \\
\text { dos escalones: formado } \\
\text { por facies y subcomarcas. }\end{array}$ & $\begin{array}{l}\text { Comarca } \\
\text { (Paraje } \\
\text { complejo) }\end{array}$ & $\begin{array}{l}\text { Coincide frecuentemente con una mesoforma } \\
\text { del relieve (o con partes de la mesoforma } \\
\text { con muchos elementos), caracterizada por la } \\
\text { asociación de regímenes de humedad, de rocas } \\
\text { formadoras de suelos, de tipos de suelos y } \\
\text { biocenosis. }\end{array}$ \\
\hline $\begin{array}{l}\text { Paisaje de estructura } \\
\text { de muchos escalones: } \\
\text { formado por comarcas y } \\
\text { subcomarcas. }\end{array}$ & Localidad & $\begin{array}{l}\text { Coincide con un determinado complejo de } \\
\text { mesoformas del relieve (positivas y negativas) } \\
\text { en los límites de una misma región, con similar } \\
\text { régimen de humedad, asociación particular } \\
\text { litológica, y un complejo o asociaciones de tipos } \\
\text { de suelos y de biocenosis. }\end{array}$ \\
\hline $\begin{array}{l}\text { Paisaje de una estructura } \\
\text { compleja de muchos } \\
\text { escalones: compuesta por } \\
\text { localidades, comarcas, } \\
\text { subcomarcas y facies } \\
\text { que forman asociaciones } \\
\text { espaciales características }\end{array}$ & Región & $\begin{array}{l}\text { Caracterizada por un fundamento geológico } \\
\text { homogéneo y de una misma edad en los límites } \\
\text { de una estructura geológica local, un mismo } \\
\text { tipo de relieve y un mismo clima. Se forma por } \\
\text { la asociación de suelos y biocenosis, que se } \\
\text { encuentran en dependencia directa de la carga de } \\
\text { hábitats locales y de configuraciones espaciales } \\
\text { que corresponden con la estructura morfológica } \\
\text { del territorio. }\end{array}$ \\
\hline
\end{tabular}

Fuente: Vidina (1970). 
Como resultado, a escala 1:250.000, se identificaron en el mapa un total de 138 localidades (2.726 polígonos), 216 parajes complejos (5.809 polígonos) y 455 parajes simples (10.838 polígonos) para el territorio de Michoacán, con amplio predominio de los geosistemas de génesis volcánica, aunque también están presentes geosistemas fluviales, lacustres y marino-eólicos, entre otros. Para efectos de esta investigación se utilizó la cartografía del nivel taxonómico de los parajes complejos, tomando como base para realizar los cálculos el número total de polígonos de los parajes simples. Para profundizar en la composición y estructura de los paisajes físico-geográficos de Michoacán, sugerimos consultar a Ramírez-Sánchez, et al. (2012).

\subsection{Evaluación de la heteroge- neidad geoecológica}

La heterogeneidad geoecológica se manifiesta a través de la diferenciación de la estructura horizontal de los geosistemas. La heterogeneidad geoecológica del territorio michoacano, medida por la diferenciación de sus parajes complejos, está determinada por el número de parajes simples que los integran y por la cantidad de polígonos de cada uno de estos parajes simples en su interior. Las unidades de paisajes en la tipología físico-geográfica son repetibles, por tanto, en un mapa de paisajes encontraremos numerosos polígonos de un mismo paraje complejo y en su interior, numerosos parajes simples.

Sobre la base cartográfica de los paisajes físico-geográficos del territorio michoacano, se identificaron los parajes complejos y se calculó el número de polígonos de cada paraje complejo, así como el número de parajes simples y el número de sus polígonos contenidos al interior de cada uno de ellos. Para realizar el cálculo de la heterogeneidad geoecológica del territorio, se utilizaron indicadores que explican las relaciones entre las unidades taxonómicas del paisaje utilizadas, es decir, entre los parajes simples y los parajes complejos:

a. Complejidad tipológica $(\mathrm{CC}=-$ ni/A, Snacken y Antrop, 1983): ${ }^{7}$ Calcula la repetitividad que tie-

7 Donde: ni= número de polígonos de los parajes simples al interior del paraje complejo; Nc= número de parajes simples presentes en el paraje complejo; Ncmax= número máximo de parajes simples contenidos en un paraje complejo; $A=$ área del paraje complejo. 
nen los parajes simples hacia el interior de los parajes complejos.

b. Complejidad corológica ( $\mathrm{CT}=-$ ni/Nc, Snacken y Antrop, 1983): Calcula el número de parajes simples por unidad de área, e indica el grado de complejidad en la estructura horizontal del paisaje.

c. Riqueza Relativa de paisajes ( $\mathrm{R}=\mathrm{Nc} / \mathrm{Ncmax}$, Turner, 1989): Computa el número máximo de parajes simples que pudieran contener los parajes complejos.

d. Diversidad máxima (Hmax=1$\mathrm{n}(\mathrm{Nc})$, Turner, 1989): Proporción entre la cantidad de polígonos y el número de parajes simples presentes en los parajes complejos.

e. Diversidad de McIntosh $(U=\sqrt{ } \Sigma-$ $\mathrm{n}_{\mathrm{i}}^{2}$, Mc'Intosh, 1991): Calcula los parajes complejos con mayor representatividad en una localidad. ${ }^{8}$

El cálculo de los indicadores en cada paraje complejo permitió establecer la heterogeneidad geoecoló-

8 Algunos de estos indicadores (c, d y e) se utilizan normalmente para estimar la biodiversidad biológica; en este trabajo se utilizaron para calcular la heterogeneidad geoecológica, sustituyendo el número de especies por número de parajes simples y el número de individuos por cantidad de polígonos al interior de cada paraje complejo. gica del Estado a nivel taxonómico de paraje complejo. Los valores de cada indicador de heterogeneidad se agruparon en cinco categorías mediante el método de clasificación de intervalos con rompimiento natural de Jenks, el cual minimiza la varianza de las observaciones respecto a su promedio dentro de cada grupo y maximiza la desviación entre grupos diferentes. Cada categoría con los valores ordenados descendentemente se renombró con las etiquetas: "Muy Alta", "Alta", "Media", "Baja" y "Muy Baja" heterogeneidad.

\subsection{Potencial natural para la conservación de la geodiversidad}

La heterogeneidad geoecológica es un reflejo de las potencialidades naturales de los geosistemas para la conservación de la geodiversidad, a mayor heterogeneidad, mayor potencial. Para el cálculo del potencial para la geoconservación se realizó la sumatoria de los indicadores calculados en cada paraje complejo y se dividió entre el número total de indicadores utilizados. Para esto se utilizó la siguiente formula: $\mathrm{PNCG}=\Sigma \mathrm{x}_{\mathrm{i}} / \mathrm{n}$, donde "PNCG" es una estimación del potencial natural para la conservación de la geodiversidad, " $\mathrm{x}_{\mathrm{i}}$ " 
es el número del indicador y " $n$ ” es el número total de indicadores utilizados en la sumatoria.

Una vez realizado el cálculo, se generaron cinco categorías de potencial natural para la conservación de la geoconservación mediante el rompimiento natural de Jenks y posteriormente se definieron cada una de las categorías (Tabla 2). Los cálculos de los indicadores de heterogeneidad se realizaron en el SIG ArcGis 10.2 (ESRI, 2012).

Tabla 2. Definición del potencial natural para la conservación de la geodiversidad

\begin{tabular}{|l|c|l|}
\hline $\begin{array}{c}\text { Potencial } \\
\text { Natural }\end{array}$ & Intervalo & \multicolumn{1}{|c|}{ Definición } \\
\hline Muy Alto & $37.97-75.57$ & $\begin{array}{l}\text { Muy Alta heterogeneidad geoecológica. Máximos valores de riqueza, } \\
\text { complejidad y diversidad de paisajes geográficos. }\end{array}$ \\
\hline Alto & $18.98-37.96$ & $\begin{array}{l}\text { Alta heterogeneidad geoecológica. Altos valores de riqueza, diversidad y } \\
\text { complejidad de paisajes geográficos. }\end{array}$ \\
\hline Medio & $9.29-18.97$ & $\begin{array}{l}\text { Media heterogeneidad geoecológica. Valores Medios de riqueza, } \\
\text { complejidad y/o diversidad de paisajes o combinaciones de Alta y Baja } \\
\text { heterogeneidad geoecológica. }\end{array}$ \\
\hline Bajo & $4.02-9.28$ & $\begin{array}{l}\text { Baja heterogeneidad geoecológica. Valores Bajos de riqueza, } \\
\text { diversidad y complejidad de paisajes o combinaciones de Baja y Media } \\
\text { heterogeneidad geoecológica. }\end{array}$ \\
\hline Muy Bajo & $1.06-4.01$ & $\begin{array}{l}\text { Muy Baja heterogeneidad geoecológica. Valores Bajos a Muy Bajos de } \\
\text { todos los indicadores. }\end{array}$ \\
\hline
\end{tabular}

Fuente: Elaboración propia.

\section{Resultados y Discusión}

\subsection{Heterogeneidad geoecológi- ca de Michoacán}

De acuerdo a los resultados arrojados por el cálculo de los indicadores de heterogeneidad, más del $80 \%$ de la superficie del Estado tiene de baja a muy baja complejidad corológica y tan solo presenta 3\% entre las categorías de alta y muy alta complejidad. Estas últimas se presentan en pequeñas superficies, distribuidas principalmente hacia el sureste del Estado, lo que nos indica que en Michoacán existe una fuerte desproporción entre las unidades tipológicas (pocas) y el área que ocupan (grande), en gran parte del territorio.

Caso contrario lo muestra la complejidad tipológica, cerca del $25 \%$ del área muestra una alta a muy alta complejidad, categoría que se distribuye principalmente hacia el norte y el noroeste del Estado (Me- 
seta Purépecha y Bajío Michoacano). Lo que señala que en esta parte del Estado se presentan unidades con gran número de polígonos en relación a las clases tipológicas que las componen. Asimismo, gran proporción de la superficie del Estado se encuentra en las categorías de baja y media complejidad tipológica con $35 \%$ y $30 \%$ respectivamente, indicando que en tales casos aumenta la equitatividad entre estas variables.

En lo que se refiere a la riqueza relativa de paisajes, $70 \%$ de la superficie está dentro de las categorías de media, alta y muy alta riqueza. Esto indica que la mayoría de los parajes complejos poseen hacia su interior un elevado número de parajes simples, en relación al número máximo de parajes simples posibles de existir en un paraje complejo; lo cual concuerda ampliamente con la gran variedad orográfica que presenta el Estado, y que hace propicia la elevada cantidad de unidades tipológicas.

La diversidad máxima muestra que más del $75 \%$ de la superficie está distribuida en las categorías de muy baja, baja y media, distribuyéndose hacia la porción central del Estado (zona de tierra caliente) y al sureste principalmente. Aquí se encuentran numerosas unidades con escasa composición de clases a su interior. Por el contrario, 25\% del área está cubierta por las categorías de alta y muy alta diversidad máxima, estas se encuentran hacia el noroeste y suroeste de Michoacán (Bajío Michoacano y en la Región Sierra-Costa), presentando geocomplejos compuestos por gran número de unidades inferiores. Estos últimos resultados muestran un patrón muy similar a los encontrados por Flores-Domínguez y Priego-Santander (2011), quienes aplicaron estos indicadores en la región costera del estado de Michoacán.

Por último, la diversidad de McIntosh muestra a las categorías de media y alta como las que presentan mayor superficie con 54\% del área, y se puede apreciar hacia el sureste del Estado y en la porción central de las montañas de la costa michoacana (suroeste). De la misma forma, las categorías de baja y muy baja presentan una extensión del $28 \%$ del total del área, distribuyéndose en la parte central del Estado. Finalmente, la categoría muy alta, ocupa $17 \%$ de la superficie del territorio y se localiza hacia el norte y noroeste del Estado (Meseta Pu- 
jos, ya sea por su extensión o por la cantidad de parajes simples que contienen a su interior (Figura 2). répecha y Bajío Michoacano). Esto último indica dónde existe una mayor dominancia de parajes comple-

A

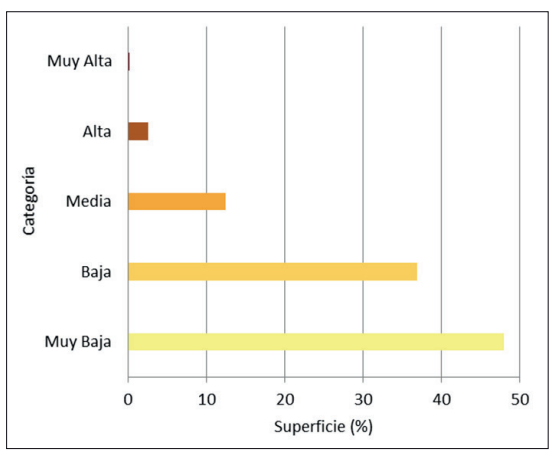

C

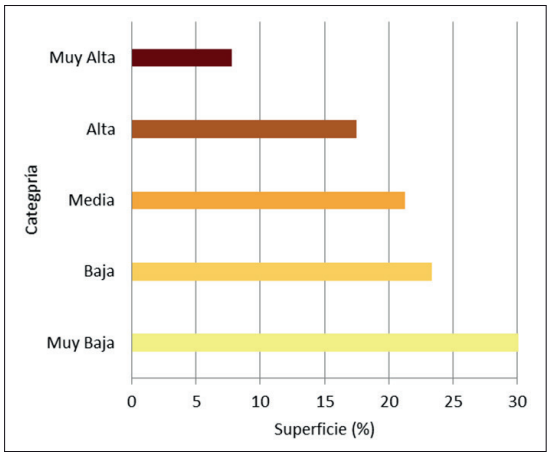

B

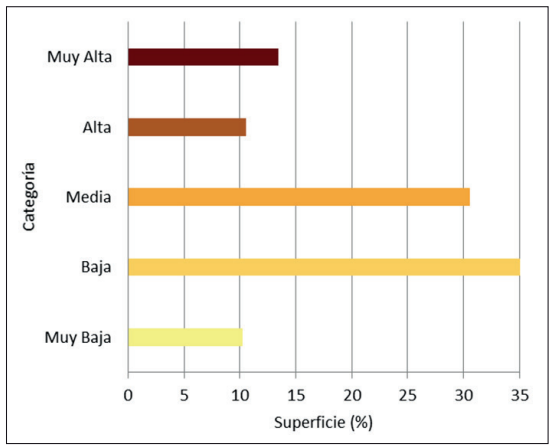

D

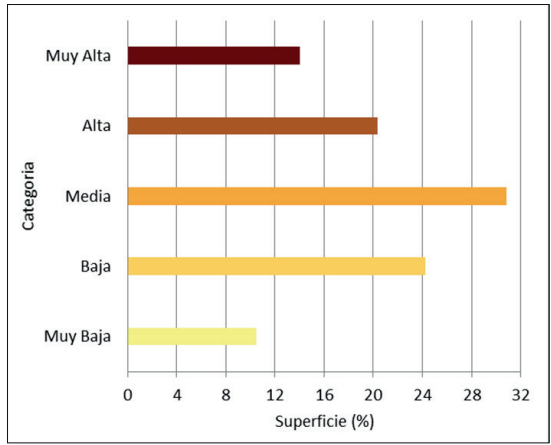

$\mathbf{E}$

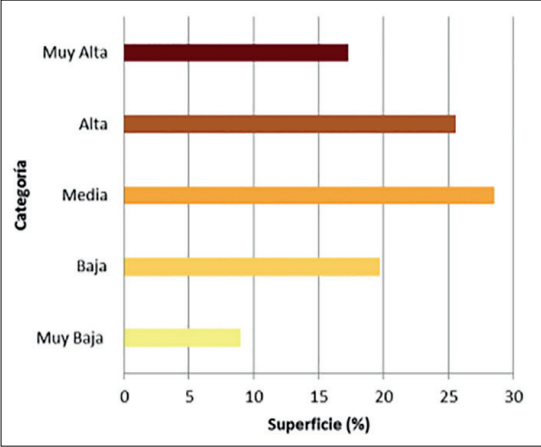

Figura 2. Indicadores de heterogeneidad: (A) Complejidad corológica; (B) Complejidad tipológica; (C) Riqueza relativa de paisajes; (D) Diversidad máxima; (E) Diversidad de McIntosh

Fuente: Elaboración propia a partir de los datos generados por los indicadores de geodiversidad. 
En la Tabla 3 se presentan los porcentajes y la superficie que preheterogeneidad en el Estado de Misentan cada uno de los índices de choacán.

Tabla 3. Indicadores de heterogeneidad geoecológica

\begin{tabular}{|l|c|c|c|c|c|c|c|c|c|c|}
\hline \multirow{2}{*}{$\begin{array}{l}\text { Indicador } \\
\text { Categoría }\end{array}$} & \multicolumn{2}{|c|}{$\mathbf{C C}$} & \multicolumn{2}{|c|}{$\mathbf{C T}$} & \multicolumn{2}{c|}{$\mathbf{U}$} & \multicolumn{2}{c|}{$\mathbf{R}$} & \multicolumn{2}{c|}{ Hmax } \\
\cline { 2 - 12 } & $\mathbf{K m}^{2}$ & $\mathbf{\%}$ & $\mathbf{K m}^{\mathbf{2}}$ & $\mathbf{\%}$ & $\mathbf{K m}^{\mathbf{2}}$ & $\mathbf{\%}$ & $\mathbf{K m}^{2}$ & $\mathbf{\%}$ & $\mathbf{K m}^{2}$ & $\mathbf{\%}$ \\
\hline Muy Baja & 5984.25 & 10.2 & 27969.27 & 47.9 & 5208.98 & 8.9 & 5986.16 & 10.2 & 17573.73 & 30.1 \\
\hline Baja & 20458.42 & 35.0 & 21503.49 & 36.8 & 11484.75 & 19.6 & 14270.62 & 24.4 & 13626.28 & 23.3 \\
\hline Media & 17828.93 & 30.5 & 7276.49 & 12.4 & 16616.71 & 28.5 & 17998.28 & 30.8 & 12399.85 & 21.2 \\
\hline Alta & 6185.74 & 10.6 & 1514.13 & 2.6 & 14917.34 & 25.5 & 11877.07 & 20.3 & 10182.20 & 17.4 \\
\hline Muy Alta & 7847.30 & 13.4 & 41.25 & 0.07 & 10076.83 & 17.2 & 8172.48 & 14.0 & 4522.55 & 7.7 \\
\hline Total & 58304.63 & 100 & 58304.63 & 100 & 58304.63 & 100 & 58304.63 & 100 & 58304.63 & 100 \\
\hline
\end{tabular}

$\mathrm{CC}=$ Complejidad corológica; $\mathrm{CT}=$ Complejidad Tipológica; $\mathrm{U}=$ Diversidad de McIntosh; $\mathrm{R}=$ Riqueza Relativa de Paisajes; Hmax= Diversidad Máxima

Fuente: Elaboración propia. 2.2. Potenciales naturales para la
conservación de la geodiversidad

Como se puede apreciar en el mapa de potenciales naturales para la conservación de la geodiversidad (Figura 3), en el territorio predominan ampliamente las zonas con bajo y muy bajo potencial (45\% del territorio). Con su mayor superficie en la parte central del Estado, principalmente en el Valle de Apatzingán (Depresión del Tepalcatepec), siendo las montañas, las de mayor representación en esta categoría. En la Región de la Meseta Purépecha se encuentran los potenciales muy altos, en esta categoría se tienen a los lomeríos como las geo- formas dominantes en cuanto a superficie se refiere. Por su parte, el potencial medio abarca casi toda la Región de la Sierra-Costa, siendo las montañas las geoformas dominantes (Tabla 4). Sin embargo, no existe correspondencia exacta en la distribución de máximos valores entre los diferentes indicadores de heterogeneidad. Esto señala la elevada complejidad del territorio y debe ser considerado en la toma de decisiones sobre los usos más convenientes a cada geocomplejo. 


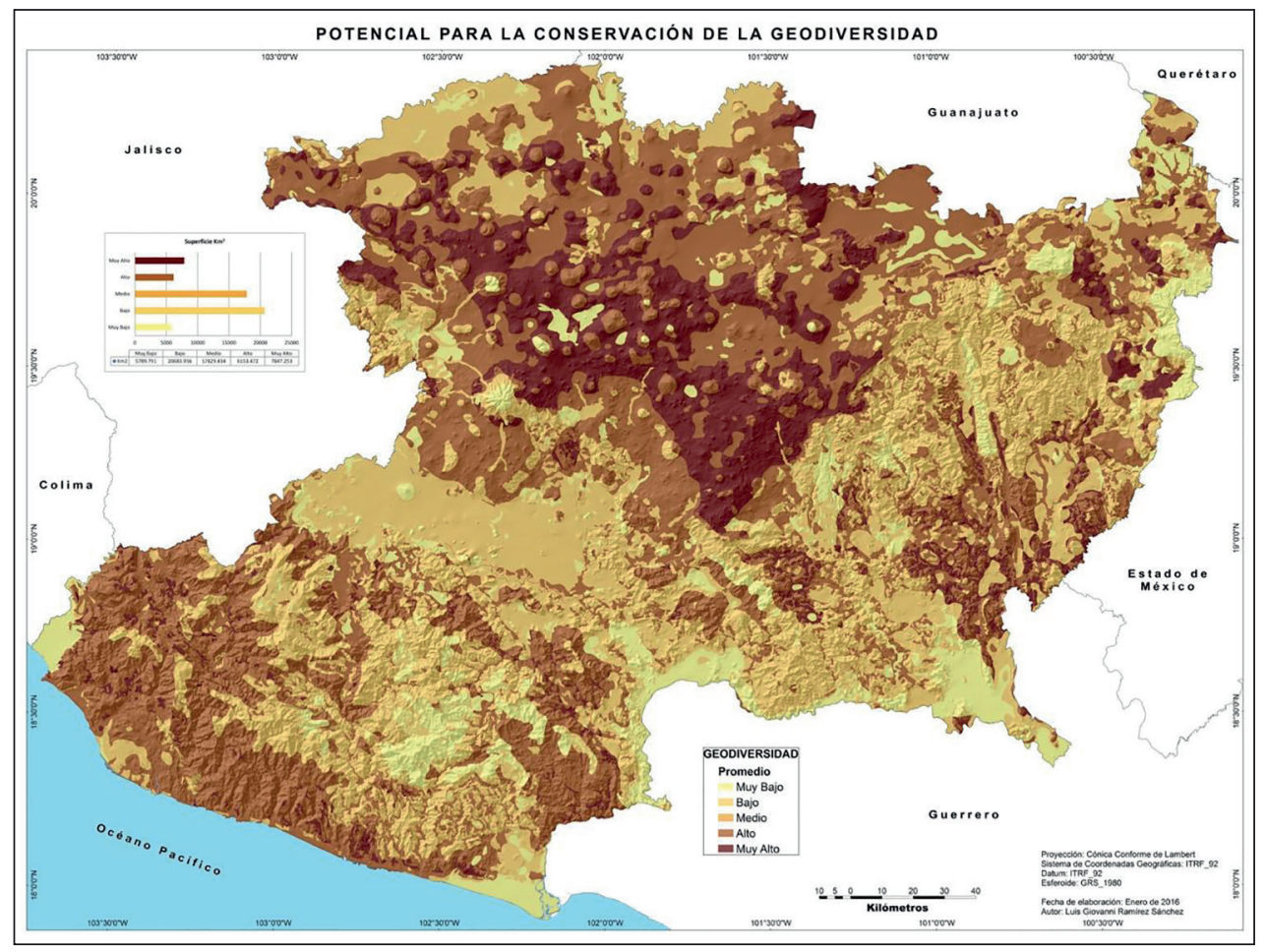

Figura 3. Potencial natural para la conservación de la geodiversidad del Estado de Michoacán

Fuente: Elaboración propia.

Tabla 4. Distribución de geoformas en los potenciales de conservación de la geodiversidad

\begin{tabular}{|c|c|c|c|c|c|c|c|c|c|c|}
\hline \multirow{4}{*}{ Geoforma } & \multicolumn{10}{|c|}{ Potencial para la conservación de la geodiversidad } \\
\hline & \multicolumn{2}{|c|}{ Muy Alto } & \multicolumn{2}{|c|}{ Alto } & \multicolumn{2}{|c|}{ Medio } & \multicolumn{2}{|c|}{ Bajo } & \multicolumn{2}{|c|}{ Muy Bajo } \\
\hline & \multicolumn{10}{|c|}{ Superficie } \\
\hline & $\mathbf{k m}^{2}$ & $\%$ & $\mathbf{k m}^{2}$ & $\%$ & $\mathbf{k m}^{2}$ & $\%$ & $\mathbf{k m}^{2}$ & $\%$ & $\mathbf{k m}^{2}$ & $\%$ \\
\hline Montañas & 1520.3 & 2.6 & 1767.6 & 3.0 & 9859.8 & 16.9 & 12047.3 & 20.6 & 2010.6 & 3.4 \\
\hline Lomeríos & 6326.9 & 10 & 1499.8 & 2.5 & 3821.5 & 6.5 & 1820.6 & 3.1 & 809.8 & 1.3 \\
\hline Planicies & 0 & 0 & 2885.9 & 4.9 & 4148.1 & 7.1 & 4747.6 & 8.1 & 2627.1 & 4.5 \\
\hline Piedemontes & 0 & 0 & 0 & 0 & 0 & 0 & 2069.6 & 3.5 & 278.9 & 0.47 \\
\hline Valles & 0 & 0 & 0 & 0 & 0 & 0 & 0 & 0 & 63.2 & 9.9 \\
\hline
\end{tabular}

Fuente: Elaboración propia. 
2.3 Algunas consideraciones sobre la geodiversdidad de Michoacán

El Estado de Michoacán posee importantes valores de geodiversidad geoecológica, los cuales no coinciden espacialmente del todo con las zonas de máxima diversidad biológica. Esto le confiere valores adicionales al área, pues, o bien prueba la necesidad de conservar nuevos paisajes, o bien sugiere que es necesario intensificar los muestreos en las zonas de máxima heterogeneidad que no reportan una elevada biodiversidad, debido a la probada relación entre ambas variables (Priego-Santander, et al., 2013). Por otra parte, estos resultados pueden ser interesantes para apoyar inferencias biogeográficas (históricas y ecológicas) considerando la escala regional de análisis, lo cual puede ser novedoso teniendo en cuenta que este enfoque no ha sido empleado para apoyar estos análisis en el territorio estatal.

De los resultados obtenidos se puede observar que la zona que presenta el mayor potencial para la conservación de la geodiversidad es la Región de la Meseta Purépecha. Mientras tanto, la Región del Valle de Apatzingán y parte del Ba- jío Michoacano presentan el menor potencial para la conservación de la geodiversidad, coincidiendo con territorios de alta asimilación socioeconómica, como actividades agropecuarias y su consecuente impacto tecnológico.

La geodiversidad de los paisajes en el estado de Michoacán determina la distribución de las variables ecológicas, sociales y económicas dentro del Estado. Al mismo tiempo, esta geodiversidad influye en el potencial del uso del suelo y la susceptibilidad a la degradación por estos usos. Como resultado de la investigación se observan evidencias suficientes para recomendar políticas, usos de conservación y protección en el territorio, estos les dan utilidad a los resultados en proyectos de ordenamiento territorial.

En muchos países en vía de desarrollo, los esfuerzos de conservación son un gran desafío debido a los déficits de información biológica y geográfica. En tales casos, este enfoque puede ayudar a mejorar la calidad de los estudios regionales, tales como: la planificación de los espacios naturales de difícil acceso con importante valor biológico (Kiester et al., 1996; Peterson et al., 2000; Rodríguez-Soto et al., 
2011); el manejo de cuencas (Morales, 2006); y la evaluación del potencial natural para la conservación y ecoturismo (Flores-Domínguez y Priego-Santander, 2011).

Existen evidencias suficientes para recomendar políticas y usos de conservación, restauración, y protección en el territorio. Sin embargo, es necesario calcular otros potenciales naturales (agrícolas, pecuarios, turísticos, etc.) para lograr una propuesta sólida de ordenamiento ecológico. Además, este trabajo no incluyó el estudio de características socioeconómicas y culturales, las cuales no pueden ser excluidas en un trabajo más integral.

\section{Conclusiones}

El enfoque empleado permitió conocer la distribución del potencial natural para la conservación de la geodiversidad en el estado de Michoacán, México, el cual se destaca por el elevado contraste entre las variables de heterogeneidad.

Michoacán se caracteriza por la poca coincidencia espacial entre los valores de heterogeneidad geoecológica, o sea: las áreas con mayor complejidad no suelen coincidir con las zonas de mayor riqueza y diversidad de paisajes. No obs- tante, más de la mitad del territorio estatal se destaca por sus elevados valores de riqueza y diversidad de paisajes, lo cual sugiere la necesidad de considerar este hecho en los proyectos de ordenación territorial. La Meseta Purépecha y la Región Sierra-Costa son las zonas que acumulan el potencial más elevado para la conservación de la geodiversidad. No obstante, otras áreas con alto potencial, y que no reportan correspondencia con elevada biodiversidad, serían zonas interesantes para profundizar los inventarios biológicos debido a la elevada correlación entre ambas variables. Además, considerando la escala regional de este análisis, estos resultados pueden apoyar inferencias biogeográficas en una dirección no explorada hasta el momento en el territorio estatal.

La cartografía de los potenciales naturales para la conservación de la geodiversidad puede apoyar las investigaciones aplicadas de ordenación territorial, sobre todo en áreas con déficit de información biológica. No obstante, debe tenerse en cuenta que esta investigación no considera otros factores socioeconómicos, los cuales son imprescindibles para una acertada planificación territorial. 


\section{Referencias}

Aguirre-López, R. (2010). Unidades campesinas de paisaje: estudio de caso en el ejido Nexpa, Michoacán. [Tesis de Maestría en Geografía]. Posgrado en Geografía-CIGA, UNAMMorelia.

Alexandrowicz, Z. \& Kozlowski, K. (1999). From Selected Geosites to Geodiversity Conservation. Polish Example of Modern Framework. In D. Barettino, M. Vallejo, \& E. Gallego. (Eds.), Towards the Balanced Management \& Conservation of the Geological Heritage in the New Milenium (pp. 52-54). Madrid: Sociedad Geológica de España.

Antrop, M. (2007). Reflecting upon 25 Years of Landscape Ecology. Landscape Ecology, (22), 1441-1443. doi:10.1007/s10980- 007-9170-y

Bastian, O. (2001). Landscape Ecology-towards a Unified Discipline? Landscape Ecology, (16), 757-766. doi:10.1023/A:1014412915534.

Boone, R. B. \& Krohn, W. B. (2000). Predicting Broad-scale Occurrences of Vertebrates in Patchy Landscapes. Landscape Ecology, (15), 63-74. doi:10.1023/A:1008169411935.

Bowler, D. E. \& Benton, T. G. (2005). Causes and Consequences of Animal Dispersal Strategies: Relating Individual Behavior to Spatial Dynamics. Biological Reviews, (80), 205-225. doi:10.1017/S1464793104006645

Brilha, J. (2016). Inventory and Quantitative Assessment of Geosites and Geodversit Sites: A Review. Geoheritage. 8, 119-134. doi: 10.1007/s12371-014-0139-3.

British Geological Survey. (2014). Why are Geodiversity Conservation Issues Important to Councilors? Recuperdo de http://www.bgs.ac.uk/planning4minerals/Geodiversity_1.htm

Burek, C. V. \& Prosser, C. D. (2008). The History of Geoconservation: An Introduction. Geological Society, (300), 1-5. doi: 10.1144/SP300.1

Cruz-García, L. M., et al. (2015). Applying Ecological Diversity Indices with Ecosystem Approach at Ecoregional Level and Prioritizing the Decree of New Protected Natural Areas. Interciencia, 40(3). 179-184.

Dóniz-Páez, J. y Becerra-Ramírez, R. (2015). Geoturismo en volcanes litorales del sur de Tenerife: Montaña Amarilla, Montaña Roja y Montaña Escachada. En III jornadas de Historia del sur de Tenerife (pp. 221-233). Arona: Llanoazur.

Dowling, R. (2013). Global Geotourism - An Emerging Form of Sustainable Tourism. Czech Journal of Tourism, 2(2), 59-79. doi: 10.2478/cjot-2013-0004 
ESRI. (2012). ArcGIS Desktop. DR. ESRI Inc. CA, USA.

Flores-Domínguez, A. D. y Priego-Santander, A. G. (2011). Zonificación funcional ecoturística de la zona costera de Michoacán, México a escala 1:250.000. Revista Geográfica de América Central, (2), 1-15.

Forman, R. T. (1995). Land mosaics: the ecology of landscape and regions. Cambridge: Cambridge University Press.

Ganzei, K. S. \& Ivanov, A. N. (2012). Landscape Diversity of the Kuril Islands. Geography and Natural Resources, 33(2), 142-148. doi:10.1134/S1875372812020072

García-Sánchez, A., Miller, A., Chamine, H., y Saiz-Jiménez, C. (2015). Estudio geomicrobiológico del patrimonio subterráneo portugués: depósitos de manganeso en la mina Das Aveleiras (Braga). Estudio y Conservación del Patrimonio Cultural. Actas Málaga.

Haines-Young, R. (1999). Landscape Pattern: Context \& Process. In J. A. Wiens \& M. R. Moss. (Eds.), Issues in Landscape Ecology (pp. 33-37). Snowmass, CO: 5th IALE-World Congress.

Hasse, G. (1986). Theoretical and Methodological Foundations of Landscape Ecology [Abstract of Lecture]. International Training Course. Institute of Geography and Geoecology, GDR Academy of Science, Leipzig, pp. 4-7.

Huston, M. A. (2014). Disturbance, Productivity, and Species Diversity: Empiricism vs. Logic in Ecological Theory. Ecology, 95(9), 2382-2396. doi:10.1890/13-1397.1

INEGI. (2001). Marco Geoestadístico Municipal. Aguascalientes, México.

INEGI-DGG. (1999). Superficie de la República Mexicana por Estados. Instituto Nacional de Estadística Geografía e Informática. Recuperado de http://www.inegi.org.mx/est/ contenidos/espanol/sistemas/Aee99/info/mic/c16_01.pdf

Kiester, A. R., et al. (1996). Conservation prioritization using GAP data. Conservation Biology, (10), 1332-1342.

Kotliar, N. B. \& Wiens, J. A. (1990). Multiple Scales of Patchiness \& Patch Structure: A Hierarchical Framework for the Study of Heterogeneity. Oikos, (59), 253-260. doi: $10.2307 / 3545542$

Kozlowski, S. (2004). Geodiversity. The concept \& scope of geodiversity. Polish Geological Review, (52), 833-837. 
Lavrinenko, I. A. (2012). Landscape Diversity of Specially Protected Natural Territories of Nenets Autonomous Okrug. Geography and Natural Resources, 33(1), 37-44. doi: 10.1134/ S1875372812010064

Li, H. \& Reynolds, J. F. (1995). On definition \& quantification of heterogeneity. Oikos, (73), 280-284

Lozano, G. (2015). Geodiversidad: cuantificación y distribución en la provincia de Segovia. En A. Hilario, M. Mendia, M. Monge, E. Fernández, J. Vegas y A. Belmonte. (Eds.). Patrimonio geológico y Geoparques, avances de un camino para todos. Cuadernos del Museo Geominero [No. 18]. Madrid: Instituto Geológico y Minero de España.

Mateo, J. (2002). Geoecología de los Paisajes: Bases para la Planificación y Gestión Ambiental. La Habana: Universidad de La Habana; MES.

Mateo, J. (2007). Geografia de los Paisajes. Primera Parte: Paisajes Naturales. La Habana: Universidad de La Habana; Ministerio de Educación Superior.

Matthews, T. (2014). Integrating Geoconservation and Biodiversity Conservation: Theoretical Foundations and Conservation Recommendations in a Europe Union Context. Geoheritage, (6), 57-70. doi:10.1007/s12371-013-0092-6

McGarigal, K. \& Marks, M. (1995). Spatial Pattern Analysis Program for Quantifying Landscape Structure. Portland: Pacific Northwest Research Station.

McIntosh, R. P. (1991). Concept \& Terminology of Homogeneity \& Heterogeneity. In J. Kolasa and S. T. A. Pickett. (Eds.), Ecological Heterogeneity (pp. 24-26). New York: SpringerVerlag.

Medina, W. (2015). Importancia de la Geodiversidad. Método para el inventario y valoración del Patrimonio geológico. Serie Correlación Geologica, 31(1), 57-72.

Merino, G., Bahamonde, J., Fernández, L. y Colmenero, J. (2015). Puntos de interés geológico en el sector oriental del Parque Natural de Fuentes Carrionas-Fuente Cobre (Norte de Palencia, Cordillera Cantábrica). Sociedad Geológica de España, Geogaceta, (58), 139142.

Milne, B. T. (1991). Heterogeneity as a Multiscale Characteristics of Landscape Studies. In J. Kolasa \& S. T. A. Pickett (Eds.). Ecological Heterogeneity (pp. 69-84). New York: Springer-Verlag.

Morales, H. (2006). Evaluación de la heterogeneidad de los paisajes y su relación con la distribución de la biodiversidad en la cuenca Lerma-Chapala, México [Tesis de Maestría en Geografía]. Posgrado en Geografía-CIGA, UNAM-Morelia.

\begin{tabular}{l|l}
\hline 340 & Luis G. Ramírez S., Ángel G. Priego S., Manuel Bollo M. y Danays del Carmen Castelo A.
\end{tabular} 
Morgan, J. L. \& Gergel, S. E. (2010). Quantifying Historic Landscape Heterogeneity from Aerial Photographs using Object-based Analysis. Landscape Ecology, (25), 985-998. doi: 10.1007/s10980-010-9474-1.

Moss, M. (2001). Preamble. In D. van der Zee \& I. S. Zonneveld. (Eds.), Landscape Ecology Applied in Land Evaluation (pp. ix-xx). IALE: Development \& Conservation.

Opdam, P., Verboom, J. \& Pouwels, R. (2003). Landscape Cohesion: An Index for the Conservation Potential of Landscape for Biodiversity. Landscape Ecology, (18), 113-126. doi:113-126.10.1023/A:1024429715253

Palacio, P. (2013). Geositios, geomorfositios y geoparques: importancia, situación actual y perspectivas en México. Investigaciones geográficas, (82), 24-37.

Peng, R., Yi, F. \& Zhao, X. (2013). Spatiotemporal Patterns in a Reaction-diffusion Model with the Degn-Harrison Reaction Scheme. Journal of Differential Equations, (254), 2465-2498. doi:10.1016/j.jde.2012.12.009

Peterson, A. T., Egbert, S., Sánchez-Cordero, V. \& Price, K. (2000). Geographic Analysis of Conservation Priority: Endemic Birds and Mammals in Veracruz, Mexico. Biological Conservation, (93), 85-94.

Plexida, S., Sfougaris, A., Ispikoudis, I. and Papanastasis, V. (2014). Selecting Landscape Metrics as Indicators of Spatial Heterogeneity- A comparison among Greek Landscapes. International Journal of Applied Earth Observation and Geoinformation, (26), 26-35. doi:10.1016/j.jag.2013.05.001

Poggiale, J. C., Auger, P., Ne'rini, D., Mante', C. \& Gilbert, F. (2005). Global Production Increased by Spatial Heterogeneity in a Population Dynamics Model. Acta Biotheoretica, 53(4), 359-370. doi:10.1007/s10441-005-4892-3

Priego-Santander, A. G., Campos, M., Bocco, G. \& Ramírez-Sánchez, L. G. (2013). Relationship Between Landscape Heterogeneity and Plant Species Richness in the Mexican Pacific Coast. Applied Geography, (40), 171-178. doi:10.1016/j.apgeog.2013.02.013

Ramírez-Sánchez, L., G. (2009). Evaluación de tierras para el cultivo del aguacate de acuerdo con el conocimiento local del paisaje en la región del Pico de Tancítaro, Michoacán [Tesis de Maestría en Geografía]. Posgrado en Geografía-CIGA; UNAM-Morelia.

Ramírez-Sánchez, L. G., Priego-Santander, A. G. y Bollo-Manent, M. (2012). Paisajes FísicoGeográficos del Estado de Michoacán. Centro de Investigaciones en Geografía Ambiental, UNAM [Marco atípico, edición digital, escala 1:250.000]. Universidad Nacional Autónoma de México, Campus Morelia, Michoacán. Recuperado de http:/www.ciga.unam.mx/ publicaciones/index.php?option $=$ com_abook\&view $=$ book\&id $=51$ :mapa-de-paisajes-demichoacan\&catid=12: coleccionesciga $\$$ Itemid $=16$ 
Rodríguez-Soto, C., et al. (2011). Predicting Potential Distribution of the Jaguar (Panthera onca) in Mexico: Identification of Priority Areas for Conservation. Diversity and Distribution, (17), 350-361.

Rowe, J. S. (1995). Eco-Diversity, the Key to Biodiversity. In A protected areas gap analysis methodology (pp. 2-9). WWF Canada Discussion Paper.

Scheiner, S. M. (1992). Measuring Pattern Diversity. Ecology, (73), 1860-1867.

Schippers, P., et al. (2015). Landscape Diversity Enhances the Resilience of Populations, Ecosystems and Local Economy in Rural Areas. Landscape Ecology, (30), 193-202. doi: 10.1007/s10980-014-0136-6

Sharples, C. (2002). Concepts \& Principles of Geoconservation. Tasmanian Parks \& Wildlife Service Website. Recuperado de http://dpipwe.tas.gov.au/Documents/geoconservation.pdf

Silva J., Rodrigues C. y Pereira, D. (2014). Mapping and Analysis of Geodiversity Indices in the Xingu River Basin, Amazonia, Brazil. Geoheritage, (7), 337-350. doi:10.1007/s12371014-0134-8.

Snacken, F. \& Antrop, M. (1983). Structure \& Dynamics of Landscape System. In Landscape Synthesis, Geoecological Foundations of Complex Landscape Management (pp. 10-30). Bratislava: Veda.

Stewart, A., John, E. \& Hutchings, M. (2000). The World is Heterogeneous: Ecological Consequences of Living in a Patchy Environment. In J. M. Hutchings, E. A. John and A. J. A. Stewart. (Eds.), The Ecological Consequences of Environmental Heterogeneity (pp.18). London: Blackwell Science.

Termorshuizen, J. W. \& Opman, P. (2009). Landscapes Services as a Bridge between Landscape Ecology and Sustainable Development. Landscape Ecology, (24), 1037-1052 doi:10.1016/j. ecolind.2013.01.037

Turner, M. G. (1989). Landscape ecology: The Effect of Pattern on Process. Annual Review of Ecology and Systematics, (20), 171-197 doi:10.1146/annurev.es.20.110189.001131

Turner, M. G. (2005). Landscape Ecology in North America: Past, Present, and Future. Ecology, (86), 1967-1974. doi:10.1890/04-0890

Turner, M. G., Donato, D. \& Romme, W. (2014). Consequences of Spatial Heterogeneity for Ecosystem Services in Changing Forest Landscapes: Priorities for Future Research. Landscape ecology, (28), 1081-1097. doi:10.1007/s10980-012-9741-4

Turner, M. G., Gardner, R. H. \& O’Neill, R. V. (2001). Landscape Ecology in Theory \& Practice. Pattern \& Process. New York: Springer-Verlag. 
Uezu, A., Metzger, J. P. \& Vielliard, J. M. E. (2005). Effects of Structural and Functional Connectivity and Patch Size on the Abundance of Seven Atlantic Forest Bird Species. Biological Conservation, (123), 507-519. doi:10.1016/j.biocon.2005.01.001

Yi, F., Wei, J. \& Shi, J. (2009). Bifurcation \& Spatiotemporal Patterns in a Homogeneous Diffusive Predator-prey System. Journal of Differential Equations, (246), 1944-1977. doi:10.1016/j.jde.2008.10.024

Zaragoza, A., Peters, R., E, Bollo, M. y Hernández, J. R. (2013). Áreas prioritarias de Geoconservación de la biodiversidad en la península de Baja California, México. Journal of Latin American Geography, 13(2),7-31. doi:10.1353/lag.2013.0036. 
Recepción: 23 de mayo de 2016 Evaluación: 9 de septiembre de 2016

Aprobación: 26 de octubre de 2016 